\title{
Surdosage accidentel de méthotrexate
}

\begin{abstract}
Nous vous prions d'adresser le formulaire d'annonce d'effets indésirables à un centre régional de pharmacovigilance. Ce formulaire peut être téléchargé sur le site web de Swissmedic (www.swissmedic.ch $\rightarrow$ Accès direct $\rightarrow$ Annonces d'effets indésirables $\rightarrow$ Pharmacovigilance), commandé auprès de Swissmedic (tél. 03132202 23) où être détaché du Compendium Suisse des Médicaments, où il est inséré en dernière page.
\end{abstract}

Julia Abegglen, Olga Frank, Marc-Anton Hochreutener, Rudolf Stoller

1 Vous trouverez les préparations autorisées en Suisse à cette adresse: www.kompendium.ch

Correspondance:

Dr méd. Julia Abegglen, Medical Reviewer Swissmedic

julia.abegglen[at]swissmedic.ch
Eu égard aux cas d'intoxication graves ayant pour partie eu une issue fatale survenus suite à l'administration/prise journalière de la dose hebdomadaire de méthotrexate, il est demandé aux professionnels de la santé de veiller à fournir aux patients une information exhaustive lors de la mise en place d'un nouveau traitement. Dès l'apparition de signes d'un possible surdosage, tels qu'une inflammation muqueuse/ stomatite, anémie, leucopénie, thrombocytopénie ou insuffisance rénale sévère, il est nécessaire de vérifier la fréquence de la prise du médicament.

Autorisé depuis 1964, le méthotrexate est un cytostatique de la famille des dérivés de l'acide folique, qui inhibent la dihydrofolate réductase et, partant, la division cellulaire. Il est souvent utilisé sous forme de comprimés pour traiter la polyarthrite rhumatoïde ou le psoriasis. Dans cette indication, le méthotrexate ne doit être pris qu'une fois par semaine.

Mais des cas de surdosage accidentel avec intoxication très grave ayant pour certains eu une issue fatale ont été régulièrement rapportés en Suisse et au plan international.

Il est important de rappeler que des intoxications peuvent se produire même si la posologie est correcte, et notamment en cas de détérioration de la fonction rénale secondaire, par exemple, à une déshydratation (diarrhée intercourante) ou à la prise d'AISN. Le risque est particulièrement important pour les patients dont la fonction rénale était amoindrie avant le début du traitement (par ex. personnes âgées).

Au cours des dix dernières années, 13 cas de surdosage accidentel ont été annoncés en Suisse (cas isolés d'administration aussi par voie parentérale; nombre total d'annonces relatives au méthotrexate: 466), dans lesquels le méthotrexate a été par erreur prescrit, administré au patient ou pris par le patient lui-même une fois par jour au lieu d'une fois par semaine. Ceuxci étaient répartis de manière régulière sur ces années, si bien qu'ils n'étaient pas très fréquents. En revanche, leurs conséquences étaient graves et 3 des 13 patients sont décédés très probablement en raison du surdosage. Aussi est-il d'autant plus important que les spécialistes soient conscients du problème du surdosage accidentel et qu'ils informent et surveillent dûment leurs patients. Dès l'apparition de signes d'un possible surdosage, tels qu'une inflammation muqueuse/ stomatite, anémie, leucopénie, thrombocytopénie ou insuffisance rénale aiguë, il est impératif de vérifier la fréquence de la prise du médicament. Certes, les informations professionnelles et destinées aux patients des préparations à base de méthotrexate [1] mettent en garde contre le risque de décès en cas de prise quotidienne de la dose hebdomadaire, mais tous les patients ne lisent pas les notices de médicament.

La Fondation pour la Sécurité des Patients a rédigé en collaboration avec plusieurs experts et sociétés savantes et publié une Quick Alert (alerte rapide) intitulée «Intoxication au méthotrexate à éviter les surdosages par voie orale», qui a pour but de sensibiliser les professionnels de santé et de leur donner des recommandations pratiques pour éviter les intoxications par voie orale.

Ces recommandations portent sur les étapes qui se situent en amont du traitement (par ex. commande/ livraison, prescription) et pendant celui-ci. Ainsi, il est conseillé d'utiliser des listes de contrôle pour la prescription de méthotrexate, de tenir compte des facteurs de risque tels que l'insuffisance rénale, la détérioration de la fonction hépatique, les troubles de la fonction pulmonaire, etc. et de s'assurer de l'absence d'interaction avec une co-médication préexistante. Quant aux patients, ils doivent eux-mêmes impérativement être informés des particularités de la prise hebdomadaire et recevoir des documents d'information rédigés à leur intention sur les risques et les signes d'intoxication et sur la manière dont il leur faut le cas échéant réagir. Ces recommandations tiennent également compte de la problématique lors des transitions, par exemple lors du passage d'un traitement stationnaire à un traitement ambulatoire. Afin de garantir la continuité du traitement à ce moment précis, il est essentiel d'assurer une gestion complète de la sortie, ce qui inclut notamment un plan de médication clair et actuel, la mention obligatoire de l'indication pour le traitement de base par de faibles doses de méthotrexate, de la dose à prendre, de l'intervalle entre deux prises et du jour de la semaine où le médicament doit être pris. Par ailleurs, pour éviter toute confusion sur le jour ou sur le moment de la journée où il faut prendre le médicament, il est interdit d'utiliser des abréviations («ma.» pourrait être interprété comme «matin» au lieu de «mardi», par exemple).

Vous trouverez les recommandations détaillées dans la Quick Alert $n^{\circ} 28$ de la Fondation pour la Sécurité des Patients, qui a été mise en ligne sur le site www.patientensicherheit.ch où elle peut être lue et téléchargée. 